\title{
Algorithmic Graph Minors and Bidimensionality
}

\author{
Erik D. Demaine \\ MIT Computer Science and Artificial Intelligence Laboratory \\ edemaine@mit.edu
}

\begin{abstract}
Graph Minor Theory, developed by Robertson and Seymour over two decades, provides powerful structural results about a wide family of graph classes (anything closed under deletion and contraction). In recent years, this theory has been extended and generalized to apply to many algorithmic problems. Bidimensionality theory is one approach to algorithmic graph minor theory. This theory provides general tools for designing fast (constructive, often subexponential) fixed-parameter algorithms, kernelizations, and approximation algorithms (often PTASs), for a wide variety of NP-hard graph problems for graphs excluding a fixed minor. For example, some of the most general algorithms for feedback vertex set and connected dominating set are based on bidimensionality. Another approach is "deletion and contraction decompositions", which split any graph excluding a fixed minor into a bounded number of smalltreewidth graphs. For example, this approach has led to some of the most general algorithms for graph coloring and the Traveling Salesman Problem on graphs. I will describe these and other approaches to efficient algorithms through graph minors.
\end{abstract}

